The University of Southern Mississippi

The Aquila Digital Community

Faculty Publications

$1-2016$

\title{
Improving Treatment of Neurodevelopmental Disorders: Recommendations Based on Preclinical Studies
}

Judith R. Homberg

Radboud University Nijmegen

Evan J. Kyzar

University of Illinois

Adam Michael Stewart

ZENEREI Research Center

Michael Nguyen

ZENEREI Research Center

Manoj K. Poudel

ZENEREI Research Center

See next page for additional authors

Follow this and additional works at: https://aquila.usm.edu/fac_pubs

\section{Recommended Citation}

Homberg, J. R., Kyzar, E. J., Stewart, A. M., Nguyen, M., Poudel, M. K., Ecchevaria, D. J., Collier, A. D., Gaikwad, S., Klimenko, V. M., Norton, W., Pittman, J., Nakamura, S., Koshiba, M., Yamanouchi, H., Apryatin, S. A., Scattoni, M., Diamond, D. M., Ullmann, J. F., Parker, M. O., Brown, R. E., Song, C., Kalueff, A. V. (2016). Improving Treatment of Neurodevelopmental Disorders: Recommendations Based on Preclinical Studies. Expert Opinion on Drug Discovery, 11(1), 11-25.

Available at: https://aquila.usm.edu/fac_pubs/17547

This Article is brought to you for free and open access by The Aquila Digital Community. It has been accepted for inclusion in Faculty Publications by an authorized administrator of The Aquila Digital Community. For more information, please contact Joshua.Cromwell@usm.edu. 


\section{Authors}

Judith R. Homberg, Evan J. Kyzar, Adam Michael Stewart, Michael Nguyen, Manoj K. Poudel, David J.

Ecchevaria, Adam D. Collier, Siddharth Gaikwad, Viktor M. Klimenko, William Norton, Julian Pittman, Shun Nakamura, Mamiko Koshiba, Hideo Yamanouchi, Sergey A. Apryatin, Maria Luisa Scattoni, David M.

Diamond, Jeremy F.P. Ullmann, Matthew O. Parker, Richard E. Brown, Cai Song, and Allan V. Kalueff 


\title{
Improving treatment of neurodevelopmental disorders:
}

recommendations based on preclinical studies

\begin{abstract}
Judith R. Homberg ${ }^{1 *}$, Evan J. Kyzar ${ }^{2,3 *}$, Adam Michael Stewart ${ }^{4}$, Michael Nguyen ${ }^{4}$, Manoj K. Poudel ${ }^{4}$, David J. Echevarria ${ }^{3,5}$, Adam D. Collier ${ }^{5}$, Siddharth Gaikwad ${ }^{3,6,7}$, Viktor M. Klimenko ${ }^{3,8}$, William Norton ${ }^{9}$, Julian Pittman ${ }^{10}$, Shun Nakamura ${ }^{3,11}$, Mamiko Koshiba ${ }^{3,12}$, Hideo Yamanouchi ${ }^{12}$, Sergey A. Apryatin ${ }^{13}$, Maria Luisa Scattoni ${ }^{14}$, David M. Diamond ${ }^{15,16}$, Jeremy F.P. Ullmann ${ }^{17}$, Matthew O. Parker ${ }^{18}$, Richard E. Brown ${ }^{19}$, Cai Song ${ }^{6,7}$ and Allan V. Kalueff $\mathrm{f}^{6,20,21 * *}$
\end{abstract}

${ }^{1}$ Department of Cognitive Neuroscience, Donders Institute for Brain, Cognition, and Behaviour, Radboud University Medical Centre, Nijmegen, Netherlands

${ }^{2}$ Department of Psychiatry, College of Medicine, University of Illinois at Chicago, Chicago, IL, USA

${ }^{3}$ The International Stress and Behavior Society (ISBS), Kiev, Ukraine

${ }^{4}$ ZENEREI Research Center, Slidell, LA, USA

${ }^{5}$ Department of Psychology, University of Southern Mississippi, Hattiesburg, MS, USA

${ }^{6}$ Research Institute of Marine Drugs and Nutrition, College of Food Science and Technology, Guangdong Ocean University, Zhanjiang, Guangdong, China

${ }^{7}$ Neuroscience Graduate Hospital, China Medical University Hospital, Taichung, Taiwan ${ }^{8}$ Pavlov Physiology Department, Institute of Experimental Medicine, St. Petersburg, Russia

${ }^{9}$ Department of Neuroscience, Psychology and Behaviour, University of Leicester, Leicester, UK

${ }^{10}$ Department of Biological and Environmental Sciences, Troy University, Troy, AL, USA

${ }^{11}$ Tokyo University of Agriculture and Technology, Tokyo, Japan

${ }^{12}$ Departments of Pediatrics and Biochemistry, Saitama University Medical School, Saitama, Japan

${ }^{13}$ Department of Psychology and Neuroscience, Dalhousie University, Halifax, Nova Scotia, Canada

${ }^{14}$ Institute of Nutrition RAS, Moscow, Russia 
${ }^{15}$ Department of Cell Biology and Neurosciences, Istituto Superiore di Sanita, Rome, Italy

${ }^{16}$ Depratment of Psychology, University of South Florida, Tampa, FL, USA

${ }^{17}$ J.A. Haley Veterans Hospital, Research and Development Service, Tampa, FL, USA

${ }^{18}$ Centre for Advanced Imaging, University of Queensland, Brisbane, Queensland, Australia

${ }^{19}$ School of Health Sciences and Social Work, University of Portsmouth, Portsmouth, UK

${ }^{20}$ Institute for Translational Biomedicine, St. Petersburg State University, St. Petersburg, Russia

${ }^{21}$ Institute of Chemical Technology and Institute of Natural Sciences, Ural Federal University, Ekaterinburg, Russia

* These authors have equally contributed to this study

\section{**Corresponding Author:}

Allan V. Kalueff, $\mathrm{PhD}$

Research Institute of Marine Drugs and Nutrition, College of Food Science and Technology, Guangdong Ocean University, Zhanjiang, Guangdong, China

Tel/Fax.: +1-240-328-2275 Email: avkalueff@gmail.com 


\begin{abstract}
Introduction: Neurodevelopmental disorders (NDDs) are common and severely debilitating. Their chronic nature and reliance on both genetic and environmental factors makes studying NDDs and their treatment a challenging task. Here, we discuss neurobiological mechanisms of NDDs, and present recommendations on their translational research and therapy, outlined by the International Stress and Behavior Society.
\end{abstract}

Areas covered: Common NDDs are a heterogeneous group which includes autism spectrum disorder, intellectual disability, communication/speech disorders, motor/tic disorders and attention deficit hyperactivity disorder. Various drugs currently prescribed to treat NDDs also represent a highly diverse group. Acting on various neurotransmitter and physiological systems, these drugs often lack specificity of action, and are commonly used to treat multiple other psychiatric conditions. There has also been relatively little progress in the development of novel medications to treat NDDs. Based on clinical, preclinical and translational models of NDDs, our recommendations cover a wide range of methodological approaches and conceptual strategies.

Expert opinion: To improve pharmacotherapy and drug discovery for NDDs, we need a stronger emphasis on targeting multiple endophenotypes, a better dissection of genetic/epigenetic factors or 'hidden heritability', and a careful consideration of potential developmental/trophic roles of brain neurotransmitters. The validity of animal NDD models can be improved through discovery of novel (behavioral, physiological and neuroimaging) biomarkers, applying proper environmental enrichment, widening the spectrum of model organisms, targeting developmental trajectories of NDD-related behaviors and comorbid conditions beyond traditional NDDs. While these recommendations cannot be addressed all in once, our increased understanding of NDD pathobiology may trigger innovative crossdisciplinary research expanding beyond traditional methods and concepts.

Keywords: neurodevelopmental disorders, animal models, autism, ADHD, translational research 


\section{Article Highlights:}

- Neurodevelopmental disorders (NDDs) are common and widespread psychiatric illnesses caused by aberrant brain development

- The chronic nature of NDDs and their strong reliance on both genetic and environmental factors complicates their treatment and prevention

- Drugs currently used to treat these disorders lack specificity and are often ineffective

- Here, we discuss neurobiology of NDDs and their available preclinical (experimental) animal models

- Based on these analyses, we formulate recommendations to improve NDD drug discovery and pharmacotherapy

- Improved understanding of NDD pathobiology is critical for fostering further innovative crossdisciplinary research in the field of translational neuroscience and biological psychiatry 


\section{List of abbreviations:}

ADHD - Attention Deficit/Hyperactivity Disorder

ASD - Autism Spectrum Disorder

CAM - complementary alternative medicine

CNS - central nervous system

CD - Communication Disorder

DSM - Diagnostic Statistical Manual

FDA - Federal Drug Administration

fMRI - functional magnetic resonance imaging

GxE - gene-environment (interaction)

G-E - gene-environment (correlation)

GWAS - genome-wide association study

ID - Intellectual Disability

ncRNA- non-coding RNAs

NDD - neurodevelopmental disorder

PPI - pre-pulse inhibition

PTSD - post-traumatic stress disorder

RDOCs - Research Domain Criteria

SERT - serotonin transporter

SHR - spontaneously hypertensive rat

SLD - Specific Learning Disorder

SNP - single nucleotide polymorphism

SSRI - selective serotonin reuptake inhibitor

WGS - whole-genome sequencing

WES - whole-exome sequencing 


\section{Introduction}

Neurodevelopmental disorders (NDDs) are caused by aberrant brain development resulting in cognitive, motor, language and affective deficits (Table 1) ${ }^{1}$. Common NDDs include autism spectrum disorder (ASD), social communication disorders, intellectual disability (ID), attention deficit hyperactivity disorder (ADHD), motor and tic disorders (Fig. 1a) ${ }^{1,2}$. Aberrant neural development, usually beginning during early embryogenesis, causes a lasting dysregulation of a wide range of brain processes, including neurogenesis, glia/neuronal proliferation, cell migration, synapse formation and

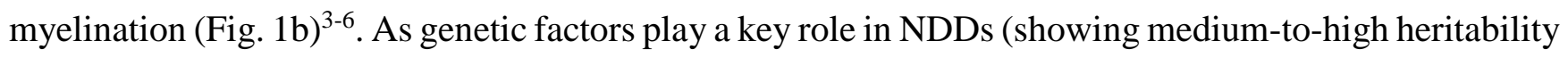
estimates; Fig. 1b), they can also be evoked by environmental factors, such as maternal influences, nutrition or early exposure to immune challenges, toxicants, psychoactive drugs and stress. In addition to clinical studies, various animal models of experimental NDDs are widely used in translational research in this field (Tables 2-4).

The growing socio-economic impact and high prevalence of NDDs require urgent attention to improving their therapy. The treatment and prevention of NDDs are complicated by their chronic nature and strong dependence on both genetic and environmental factors. With little progress in the development of novel medications for NDDs, the existing drugs often lack specificity of action, and are mainly used to treat other psychiatric conditions beyond $\operatorname{NDDs}^{7}$ (Table 3). Recognizing these important challenges, the International Stress and Behavior Society (ISBS) has established the Strategic Task Force on NDDs. Comprising international experts from different fields of biological psychiatry, the Panel comprehensively evaluated the neurobiological mechanisms, genetics, psychopharmacology and in-vivo animal models of NDDs. Based on mounting translational and preclinical evidence, the ISBS Task Force has made critical recommendations for improving pharmacotherapy of NDDs, which are summarized here.

\section{The complex genetics of neurodevelopmental disorders}

Described as a "ballet choreographed over time between the action of multiple genes, environmental and epigenetic factors" ${ }^{\prime}$, brain disorders often display complex, polygenic non-Mendelian 
genetics ${ }^{9}$. Used to identify disease-causing variants, genome-wide association studies (GWAS) utilize multiple single nucleotide polymorphisms (SNPs) maintained in linkage disequilibrium ${ }^{10}$. However, the contribution of GWAS to our understanding of brain disorders, and NDDs in particular, has been limited so far ${ }^{9}$, partially due to the small sample sizes, the difficulty of ascertaining how SNPs alter gene function, the variability of clinical NDD symptoms and the poor operationalization of these symptoms into measurable entities. The common variants uncovered by GWAS only account for a small percentage of heritability estimates, with surprisingly poor correspondence of data across studies ${ }^{10,11}$. Yet, in some cases, genome-wide approaches followed by fine-mapping may provide useful insights into the genetics of NDDs. For example, Latrophilin 3 (LPHN3) was identified as a human ADHD 'susceptibility' gene by linkage analysis of affected families ${ }^{12}$ and is now being confirmed in preclinical models of this disorder ${ }^{13}$.

Increasing evidence indicates that some NDDs (e.g., ASD and ADHD) can also be triggered by mutations in single genes ${ }^{14-17}$. These genes often contain rare mutations with $<1 \%$ of the minor allele frequency (the rate at which the less common allele occurs within a given population) ${ }^{10}$. Rare mutations include both SNPs and copy-number variants ${ }^{18}$ - deletions or duplications of chromosome segments of variable size that can affect single or multiple genes at the same time. Rare mutations can impact brain and its development in several ways. In addition to reducing gene function, disease-causing mutations may constitutively activate proteins, show dominant negative activity, or create new or abnormal biochemical functions ${ }^{19,20}$. Furthermore, a single disease gene can be mutated in multiple positions, each of which may give rise to a very different phenotype. For example, a defect in a single gene, Disrupted in Schizophrenia 1 (DISC1), can lead to different disorders, including schizophrenia (psychoses), bipolar disorder, major depression or ASD, even when patients carry the same mutation ${ }^{21}$. Therefore, for NDDs with an unknown genetic basis, it may be critical to identify causative mutations in both known and novel genes.

The non-Mendelian inheritance of some single disease-causing mutations can be explained by incomplete penetrance of the disease-causing alleles, by protective variants in the genetic background or 
by the acquisition of novel mutations. Single mutations provide an excellent entry point for studying NDDs, as they are easier to model in animals, provide construct validity for the disease and offer opportunities to uncover the underlying neural circuits ${ }^{11}$. Furthermore, comparison of the effect of multiple mutations may also highlight the general changes in brain function that lead to pathogenesis ${ }^{22}$ : just as "all roads lead to Rome", alteration of the function of many genes may ultimately affect only a few common disease-causing pathways ${ }^{23}$ (see further). However, the relative contribution of rare or common mutations to disease susceptibility is currently unclear. Rather than being caused by a single type of genetic lesion, NDDs could be caused by a combination of many. For example, single mutations can predispose patients to ADHD, while other SNP polymorphisms in the genetic background (or mutations in a second critical gene) may alter the penetrance of the disease ${ }^{11}$. Thus, a single mutation may be necessary, but not sufficient, to trigger the disorder. Such a combined model may explain the large heterogeneity of NDD symptoms and their low penetrance often observed in patients and in preclinical models ${ }^{24}$.

Since most of the rare mutations linked to NDDs affect genes active during embryonic development, even subtle changes can have far-reaching consequences for neural development and permanently alter the mature brain function ${ }^{25}$. In some cases, genetic mutations cause specific cellular phenotypes linked to neurological disorders, impairing symmetric cell division, differentiation, survival, neurite outgrowth, axon pathfinding and dendritic architecture ${ }^{26,27}$. Alternatively, mutations may cause diffuse and variable changes to brain function by influencing the outcome of random variation at a number of developmental choice points, thereby affecting the global developmental trajectory of an $\operatorname{organism}^{28}$. Such broad phenotypic changes may cause miswiring of neural circuits, leading to altered brain function and pathophysiology ${ }^{29}$. Overall, diffuse changes to brain function seem likely when considering that genes do not directly control behavior, but rather act via the formation, connection and function of neural circuits in the brain. Thus, it is perhaps predictable that many disease-causing mutations are active during embryonic development, a time when embryos may be both more plastic and more susceptible to alteration. 


\section{Recommendations of the ISBS Strategic Task Force on neurodevelopmental disorders}

Outlined here are several strategies suggested by the ISBS Panel to foster translational research into the pathogenesis and treatment of NDDs (also see Table 5). Covering different aspects of translational cross-species and multi-phenotype modeling, these recommendations are expected to improve current and future drug discovery and pharmacotherapy of NDDs.

\subsection{Focus on multiple (vs. single) endophenotypes}

While prenatal neural development is associated with cell differentiation, neuronal outgrowth and network formation (Fig. 1b), after birth, these networks are refined by the experiences of the newborn that impinge on the ongoing developmental processes. Therefore, it is insufficient to elucidate neurodevelopmental mechanisms by studying individuals with full-blown disordered phenotypes. Indeed, it has been demonstrated that NDDs are associated with susceptibility genes that are relatively upregulated in the prefrontal cortex during fetal life ${ }^{30}$. For example, genes associated with syndromic NDDs (e.g., ID and ASD), are relatively enriched in prenatal transcript abundance compared with the overall transcriptome, whereas genes associated with neurodegenerative disorders are significantly under-expressed during fetal life ${ }^{30}$. In addition, during normal prefrontal cortex development, gene splicing changes are most frequent early in development. More than $60 \%$ of all splicing changes represented a single splicing pattern reflecting preferential inclusion of gene segments potentially targeting transcripts for nonsense-mediated decay in infants ${ }^{31}$. Risk for NDDs is increased when nonsense-mediated mRNA decay becomes aberrant ${ }^{32}$. Thus, it is essential to follow developmental processes before phenotypes become overt. Some of these processes may remain sub-threshold and not present recognizable features. Even in this case, a feature appearing will not lead to a diagnosis. As a consequence, a continuum in developmental processes and associated phenotypes is expected. Longitudinal and prospective cohort studies providing information about maternal and paternal genotype and personality, prenatal factors, and genotype, brain morphology and function, life events and developmental milestones can provide important insights into this continuum of developmental processes and phenotypes that predispose to the full-blown diagnosed disorder. However, such studies require a 
large number of subjects and more budget, given clinical heterogeneity of NDDs (Fig. 1a-c). Traditionally, experimental modeling of NDDs and other brain disorders is performed by mimicking their behavioral (affective, cognitive, social, motor or reward) endophenotypes ${ }^{8,33}$. Current preclinical studies of NDDs may therefore benefit from modeling the dynamic interplay of multiple pathogenic endophenotypes (and their sensitivity to pharmacotherapy) rather than focusing on individual phenotypes or their screens. Note, however, that this approach can be complicated in longitudinal studies (revealing phenotypes and mechanisms occurring before the onset of the full-blown disorder). Thus, development of novel 'early' biomarkers or endophenotypes of NDDs, and performing both short-term and 'cliniclike' longitudinal experiments in animal models, is recommended.

\subsection{Dissect genetic and epigenetic influences}

Modern genetic sequencing tools have revealed multiple previously unknown genes linked to NDDs. Many of the sequencing studies performed to date have utilized whole-exome sequencing (WES), which focuses only on the $\sim 2 \%$ of the human genome that encodes proteins. Recently, the Encyclopedia of DNA Elements (ENCODE) project found that the non-coding regions of the genome not sequenced in typical WES play crucial regulatory functions in cellular dynamics and pathways ${ }^{75}$. In contrast, wholegenome sequencing (WGS) attempts to scan the majority of the genome for disease-linked genetic variants, and is successful in small cohorts of ASD patients ${ }^{57,76}$. While WGS poses financial challenges, this Panel recommends an increased focus on WGS, as this may lead to further discoveries of rare variants in non-coding RNAs (ncRNA) and other regulatory regions contributing to NDDs. The examination of non-coding genomic regions and ncRNA also highlights the need for detailed epigenetic analyses of NDDs. Indeed, epigenetics play a critical role in the pathogenesis of Angelman syndrome (loss of imprinting at $U B E 3 A$ ), Fragile $\mathrm{X}$ syndrome (hypermethylation of $F M R 1$ promoter caused by trinucleotide expansion), and Rett syndrome (mutation in the epigenetic enzyme $M E C P 2)^{77,78}$. Mutations in genes coding for chromatin-modifying enzymes were also recently identified in $\mathrm{ASD}^{60}$. Therefore, we call for the detailed investigation of both epigenetic markers (e.g., histone acetylation/methylation and DNA methylation) in NDDs, as well as deep sequencing analysis of genes encoding epigenetic enzymes (such 
as MeCP2, DNA methyltransferases and histone deacetylases/acetyltransferases), since mutations in these enzymes may play a role in stochastic epigenetic variation and transgenerational epigenetic effects (e.g., $\left.{ }^{79}\right)$.

\subsection{Focus on symptoms that bridge across several neurodevelopmental disorders}

The symptoms of NDDs frequently overlap (Fig. 1b), providing an opportunity to uncover common down-stream pathways that affect multiple diseases. For example, aggression is a common comorbid symptom of ASD, ADHD, schizophrenia, conduct disorder and some types of depression ${ }^{\mathbf{3 4}}$. Although heightened aggression levels can prevent treatment of other symptoms of a disease, the neural circuits that control this behavior are not well understood. Additionally, there are currently few medications that specifically target this behavior and, therefore, an urgent need for effective pharmacotherapy. Therefore, studying the genetic and neurological basis of behaviors that are present across neurodevelopmental disorders may provide insight into each separate disease. This recommendation is generally consistent with the recently proposed Research Domain Criteria (RDOCs) of the US National Institutes of Health $(\mathrm{NIH})$ to address mechanisms and phenotypic dimensions, rather than symptomatic categories, of psychiatric diagnoses ${ }^{80}$. By focusing on discrete phenotypes, rather than on whole, criteria-based NDDs, new insights may be gained that would have otherwise remained obscured.

\subsection{Address the problem of 'hidden heritability'}

The problem of 'hidden' (missing) heritability ${ }^{35}$ is highly relevant to pathobiology of NDDs, since impaired neural development often causes global behavioral and cognitive deficits. This, in turn, may mask disorder-specific phenotypes, which may then become undetectable by conventional (e.g., GWAS) analyses. Recognizing its potential importance, we call for more thorough analyses of 'hidden heritability' of NDDs in both clinical and preclinical studies. Additionally, given the importance of epigenetic factors in CNS modulation, detailed analyses of epigenetic variation ${ }^{81}$ (both inherited and random) may further contribute to the "missing heritability" of complex brain disorders, such as NDDs. For example, genetic risk for a specific NDD may be passed through the germline by either "true" 
epigenetic inheritance (i.e., an epigenetic mark that is present in the germline and inherited across multiple generations) or by mutations in epigenetic enzymes which may bias the chromatin confirmation of a patient ${ }^{36,37}$, thus increasing the risk for diagnosis of a particular NDD.

\subsection{Examine potential developmental role of neuromediators}

Serotonergic drugs, such as SSRIs, are commonly used to treat NDD symptoms (Table 3). Mounting evidence suggests that some neurotransmitters, such as serotonin, may play a dual role in the brain, acting as both classical neurotransmitters and developmental 'neurotrophic' hormones ${ }^{38}$. For example, although the genetic ablation (knockout) of serotonin transporter (SERT) in mice shares some similarities with the effects of postnatal SSRI exposure, some differences exist, including anxiety and elevated extracellular serotonin levels in SERT knockouts vs. reduced serotonin levels and anxiety in adult mice following early postnatal SSRI (escitalopram) ${ }^{39}$. Therefore, it remains unclear to what extent serotonin-mediated neurodevelopmental changes contribute to serotonin-related traits, and whether such traits are solely dependent on serotonin levels per se. The developmental phase during which serotonergic perturbations take place can play a crucial role, as during the late prenatal phase in rodents serotonergic neurons grow and migrate, whereas at the early postnatal phase brain circuits are shaped and refined ${ }^{40}$. Furthermore, it remains unclear whether and which serotonin-mediated structural neurodevelopmental changes contribute to behavioral phenotypes of NDDs. Therefore, approaches like in-utero electroporation and manipulating Sert expression during embryonic development may help clarify the postnatal structural and functional neurodevelopmental role of serotonin. Focusing on developmental processes during the postnatal period, the use of conditional knockout animals may further reveal how changes in neural development contribute to NDD-like behavioral phenotypes. For example, SSRIs used during pregnancy cause subsequent developmental delays, increased social behavioral disturbances and increased risk for $\mathrm{ASD}^{41,42}$ in children. In addition, SSRIs induce chromatin alterations, suppressing protein kinase calmodulin-dependent protein kinase II (CaMKII) transcription in the rat nucleus accumbens $^{36}$. Collectively, this suggests a larger (than traditionally recognized) role for central serotonin, as it can modulate NDDs directly as neurotransmitter, and indirectly as a neurotrophic developmental 
hormone. Investigating this emerging complexity, and its implications for pharmacotherapy of NDDs, are recognized by this Panel as an important strategic direction of research.

\subsection{Improve the validity of animal models and biomarkers}

Given their immense translational importance, we recommend the continued use of geneticallymodified animal models to investigate the neurobiology of NDDs, with a particular focus on genes involved in synaptic function and epigenetic modification ${ }^{2,60}$. However, efforts should also focus on the development of environmental epigenetic models that recapitulate early life factors and may be involved in these disorders. The combination of these preclinical models will be crucial in identifying gene $\mathrm{x}$ environment interactions important for the progression of NDDs. Notably, basic research continues to over-utilize male animals and cells ${ }^{43}$. As various NDDs demonstrate robust sex differences (Fig. 1), both sexes must be included in a balanced manner in preclinical studies to improve drug discovery. Likewise, with the growing availability of rodent or fish strains for NDD research, a few selected inbred strains are typically used for drug screening assays, to ensure a better genetic control of the experiments ${ }^{44}$. As the global human population is highly heterogeneous genetically, a more balanced use of both inbred and outbred animal strains in CNS drug discovery may lead to more valid treatment and side-effects data, reflecting 'demographic' aspects of drug action and providing important clinical insights. Finally, although biological markers are not yet approved as part of the diagnostic criteria for NDDs, they may help predict the potential disease trajectory, and support decisions for specific early therapeutic and prophylactic measures. Other well-validated physiological biomarkers relevant to NDDs include fMRI imaging and, more broadly, other electrophysiological markers (e.g., mismatch negativity) which have shown high sensitivity to $\mathrm{NDDs}^{80,81}$. We strongly emphasize the need to validate and discover new predictive markers of neurodevelopmental markers, in order to improve both drug discovery and pharmacotherapy.

\subsection{Widen the spectrum of model organisms}

Preclinical animal models are a critical tool in biological psychiatry ${ }^{45}$, including translational modeling of NDD-related phenotypes and pathogenesis. For example, rodent models of social 
interaction, communication deficits and repetitive behaviors are relevant to studying ASD, whereas rodent hyperactivity and/or inattention can parallel clinical ADHD. Although rodents are currently the most commonly used animal model in the pre-clinical research (Fig. 2), they are limited by lower throughput and costs ${ }^{45}$. Therefore, increasing the spectrum of model organisms is recognized as an important strategy in biological psychiatry, and is fully endorsed by the ISBS. This endorsement is even more based on the conceptual value of identifying core, evolutionarily conserved pathogenic mechanisms of brain disorders - which can only be achieved by modeling brain disorders across taxa ${ }^{57,59}$. For example, zebrafish (Danio rerio) are a time-efficient and low-cost model with high genetic and physiological homology to humans (Fig. 2) ${ }^{46,47}$. They also show rapid development, which beneficial for NDD models larval zebrafish display robust motor and affective behaviors ${ }^{48}$ (which can be then monitored throughout the 4-year lifespan). In adult zebrafish, group behavior (shoaling) can be a useful model of $\mathrm{ASD}^{49}$, whereas hyperlocomotion, impulsive swimming and inattention in both larval and adult zebrafish can be relevant to $\mathrm{ADHD}^{50,51}$. Chicks (Gallus gallus) also display robust social and locomotor phenotypes, recapitulating several NDD symptoms, including social deficits, accompanied by aberrant brain growth $^{52}$ and affective behaviors ${ }^{53,54}$. Likewise, non-human primates, such as common marmosets (Callithrix jacchus), represent another useful model organism with robust social and affective behaviors $^{55,56}$ highly sensitive to environmental manipulations that cause NDD-like phenotypes (e.g., ASD-like social deficits or ADHD-like pathological hyperactivity ${ }^{56}$ ). Finally, complementing vertebrate models, invertebrate models (e.g., fruit flies, Drosophila melanogaster) are also demonstrating potential for modeling some aspects of NDDs ${ }^{57-59}$. Although these models have been comprehensively evaluated in the literature (see above), and will not be discussed here in detail, increasing the spectrum of model organisms is recognized by this Panel as one of the top priorities in translational NDD research.

\subsection{Assess the role of environmental enrichment}

There is currently a growing interest in the impact of early life adversity on neural development. Less attention, however, has been paid to the effects of environmental enrichment. Rearing laboratory animals in a complex, enriched environment can stimulate the development of the CNS, aid in 
recovery of brain function following acquired injury, and improve performance on many cognitive and behavioral tasks, affording it special interest in the study of NDDs ${ }^{37,60,61}$. The potential for modulation of NDD phenotypes by enriched environments has received some attention (e.g., in particular, with respect to refinement of animal models), and evidence is emerging that the effects of some teratogen-linked NDDs can be improved by environmental enrichment ${ }^{62}$. For example, environmental enrichment reverses pre-pulse inhibition (PPI) deficits, alterations in pain sensitivity, stereotypic behavior, and social behavioral deficits in a mouse model of ASD induced by pre-natal valproate exposure $^{62}$. Furthermore, environmental enrichment can significantly improve both the physical and behavioral response to prenatal alcohol exposure in rodents ${ }^{63-65}$ and enhance cognitive performance in spontaneously hyperactive rats (SHR), a genetic model of ADHD ${ }^{66}$. Although the mechanisms of action on NDD-related phenotypes are not particularly well understood, they may include the modulating effects of environmental enrichment on brain neuroplasticity and the efficacy of dopamine receptors in the pre-frontal cortex ${ }^{67,68}$. Therefore, gaining a more thorough understanding of the role of environmental enrichment on the modulation and mediation of NDDs will be of critical importance moving forward. Additionally, the Panel emphasizes the interaction of genetic factors and environmental enrichment on the affectation of NDDs as a potential priority for further research in this field.

\subsection{Address Gene-Environment interactions vs. Gene-Environment correlations}

The Gene x Environment interactions (GxE) characterize how genotypes modify the sensitivity to environmental factors, and are widely analyzed in neuropsychiatric research. The gene-environment (G-E) correlations represent another important source of variation for complex behavioral traits, reflecting the individuals' exposure to the environment as a function of their genotype ${ }^{69}$. The understanding that environment is not a static uniform factor, but can partly depend on individual's own behavior, is an important conceptual development in modern biological psychiatry ${ }^{69}$. This concept has direct implications for NDDs and their association with environmental factors, especially those which are behaviorally modifiable (e.g., social environment). Consider, for example, parent- 
offspring and early peer-peer interactions, which represent 'early' reciprocal social interactions during neural development ${ }^{69}$. While most animal studies view the 'environment' as a static factor equally imposed on all animals in the laboratory, our 'real life' experience depends on subjects' behavior with parent, relatives or peers. For children with genetically caused poorer social skills (e.g., ASD), their peculiar mode of interactions with others can make social environment more adverse, compared to 'smoother' healthy subjects. On the one hand, such behaviorally-mediated interactions with the environment may affect neural development in a correlated (or even synergistic) manner. On the other hand, breaking this vicious pathogenetic cycle may reduce the negative impact of environmental adversity, therefore contributing to a better therapeutic outcome. The Panel recognizes the growing importance of G-E correlations in NDDs, and recommends more animal and clinical studies of reciprocal influences between environment and neural development.

\subsection{Use imaging phenotypes in the field of neurodevelopmental disorders}

Modern imaging technologies enable in vivo monitoring of NDDs and in vivo assessment of the efficacy of novel pharmacological treatments. Magnetic resonance imaging and diffusion weighted imaging are established longitudinal methodologies for identifying neuroanatomical alterations. Threedimensional brain atlases exist for a range of species and enable automatic segmentation of brain regions to identify differences in volume or white matter microstructure ${ }^{70-75}$. These imaging modalities have been widely used to study various NDDs, including ASD, ADHD and $\mathrm{ID}^{76}$. More recent functional imaging techniques have begun to identify the neural circuitry linked to the behavioral and social phenotypes relevant to NDDs. For example, manganese-enhanced magnetic resonance imaging ${ }^{77-79}$ and functional magnetic resonance imaging (fMRI) ${ }^{80,81}$ can identify abnormal circuitry, while optogenetics permits identification and precise manipulation of the circuitry related to neurological disorders ${ }^{82-84}$. In vivo imaging also plays an important role in drug discovery - e.g., as it helps to establish vital biomarkers that are necessary to determine if therapeutic candidates have elicited their targeted biological effects ${ }^{85}$. Following identification of a disease biomarker, imaging can then be used to profile drug mechanisms, efficacy, and safety. A wide range of such techniques include ultrasound, magnetic resonance 
spectroscopy, positron emission tomography, single-photon emission computed tomography, and optical imaging methods (e.g., near-infrared fluorescence microscopy $)^{86}$. Method to generate transparent brains are now also emerging ${ }^{87}$, which jointly with novel microscopic techniques enable 3D brain imaging. Each technique has different spatial resolutions and time scales, resulting in unique advantages and limitations. As a result, studies should carefully consider which technique(s) are best suited to produce desired conclusions. Overall, the Panel recognizes the value of the diversity of neuroimaging tools, as well as simultaneously analyzing physiological biomarkers and genomic/epigenetic mechanisms. We strongly believe that increased understanding of NDDs must be paralleled by advances in imaging. Imaging can play a large role in many suggestions of the Panel listed here, such as identifying similarities and differences between sexes or strains, developing canonical template spaces for new animal models, investigating the effects of environmental enrichment, and longitudinal investigations of brain development. Ultimately, by combining with existing behavioral and social paradigms, imaging methodologies will further foster translational research of NDDs.

\subsection{Increase focus on neurodevelopmental trajectories}

Many NDDs are triggered during embryonic development and their symptoms predominantly affect children or young adults. However, the majority of studies in animal models have focused on the behavior and neurobiology of fully mature adults. We recommend that research be extended to more actively include juvenile animals as well. The adolescent brain undergoes widespread developmental changes that affect axon guidance and proliferation, circuit wiring, and synaptogenesis and pruning. These structural changes are associated with the modulation of genetic and epigenetic factors. As NDDs likely affect these processes, a critical step will be to compare neuronal position, connectivity and the function of neural circuits in brains that are not yet fully mature, as well as their underlying molecular mechanisms. For example, ADHD has been linked to a delay in brain maturation and aberrant myelination, manifested as a reduced volume and aberrant microstructure of the white matter ${ }^{88,89}$. Furthermore, the effect of pharmacotherapy on brain development trajectories should also be investigated further (Fig. 3). 


\subsection{Widen the spectrum of disorders relevant to NDDs}

NDDs frequently overlap with other neuropsychiatric disorders, including anxiety, depression, schizophrenia and post-traumatic stress disorder (PTSD, Fig. 1). Therefore, expanding the spectrum of NDD-related disorders is an important strategy in translational NDD research, and can include modeling brain illnesses that are not conventional NDDs. Consider, for example, the potential link between NDDs and PTSD, as neurodevelopmental deficits can later make an adult individual more vulnerable to stress, thereby triggering or exacerbating developing PTSD after a traumatic event 90 , 91. Alternatively, depending on age, early traumas (e.g., child abuse) could potentiate neurodevelopmental deficits, which will parallel the developing PTSD symptoms. Thus, vigilant pharmacotherapy of PTSD may represent an important adjunct therapy to prevent or reduce NDDs (Fig. 1). The finding that some NDD symptoms (Table 3) and some forms of PTSD exhibit improvement following treatment with SSRIs suggests a role of the serotonergic system in these mechanisms ${ }^{92}$. Two SSRIs, paroxetine and sertraline, are currently the only FDA-approved pharmacological treatments for PTSD. However, response rates to SSRIs in PTSD patients rarely exceed $60 \%$, whereas full remission following SSRI treatment is achieved in only $20-30 \%$ cases $^{93}$. An indication of the accentuated sympathetic activity in PTSD patients is the hyperresponsivity to the administration of yohimbine, an $\alpha_{2}$-adrenergic receptor antagonist that inhibits noradrenergic autoreceptors and increases central norepinephrine activity ${ }^{94}$. Along with a generally greater baseline norepinephrine levels in PTSD patients, this indicates a critical role of this neurotransmitter in the hyperarousal component of PTSD. Based on observed hyperactivity of the noradrenergic system in PTSD, the efficacy of anti-noradrenergic drugs in treating the disorder is supported by the use of propranolol, a $\beta$-adrenergic receptor antagonist, to reduce PTSD symptoms when administered after a traumatic event or with re-experiencing a traumatic memory ${ }^{95}$. Moreover, in preclinical studies, stress consistently increases glutamate levels, inhibits glutamate uptake, increases the expression and binding of glutamate receptors and increases calcium currents. For example, the primary effect of tianeptine involves the stabilization of glutamatergic neurotransmission and the enhancement of synaptic plasticity, particularly under stress conditions ${ }^{96}$ - the two mechanisms that may bridge NDD- 
and PTSD-related pathogenesis. Taken together, this evidence suggests that examining disorders not normally considered as NDDs, such as PTSD, may provide new important insights leading to novel treatments that may be beneficial for comorbidity with, and/or target some symptoms of, NDDs.

\section{Conclusion}

In recent years, research into NDDs has revealed important information about their pathogenesis and underlying biology. However, due to the challenging and complex nature of NDDs and their shared or unique etiologies, many of the neural mechanisms involved remain unknown, resulting in a lack of effective treatment options for patients. Increasing the throughput of the translational pipeline from invitro assays to clinical trials (Fig. 3), and using mechanistic insights generated from experimental observations, will lead to novel effective, target-specific therapies available to patients suffering from NDDs.

\section{Expert Opinion}

Translational in-vivo research aims to replace symptomatic drug therapies with those based on a principled understanding of the disease causes. In relation to NDDs, this process can be presented as a translational cycle with four multidisciplinary steps (Fig 2). The first step, animal modeling, uses animals to examine cognitive, motor and social behaviors, and their underlying genetics, molecular biology, neurophysiology and anatomy. Next, therapeutic strategies step aim to design interventions based on biological findings in animal models and optimize drug safety and efficacy. The drug development step then involves optimization of lead compounds to improve drug-target specificity, bioavailability and pharmacokinetics, as well as to determine the most efficacious dose, dosing strategy and route of administration. Finally, clinical trials examine the drug's therapeutic effects as well as their behavioral and cognitive effects in humans (Fig. 2 and 3).

Careful study of brain pathology and behavior establishes how well an animal model represents human disease (Step 1). Therapeutic strategies are evaluated in animal models by measuring deficits in performance in various behavioral tasks (Step 2, Table 2). A particular therapeutic strategy may address only a subset of cognitive or motor functions, so multi-target therapies that correct distinct deficits and 
brain areas may be desirable. Drug development optimizes a therapeutic compound to improve drugtarget specificity, reduce or eliminate dangerous side effects, and determine dose and route of administration (Step 3). Finally, a lead compound enters clinical trials to test the safety and efficacy of therapeutic strategies discovered in animal models (Step 4). An approved pharmacotherapy for a NDD (see Table 3 for a partial listing of currently used and approved drugs) would reduce one or more cognitive deficits or maladaptive behaviors. Such a therapy completes the translational cycle by addressing the disease phenotype. However, animal models are often an imperfect representation of human disease or developmental disorders, and the differences between species may carry special significance for disease pathology ${ }^{97-99}$. Thus, improved characterization of animal models of NDDs requires better behavioral assays and physiological measurements. In addition, a better dissection of genetic determinants of NDDs is necessary - especially since, as already mentioned, even a single mutation can result in different CNS diseases within family pedigrees ${ }^{100}$.

New animal models developed in the future will hopefully improve the correspondence with human conditions we hope to achieve, especially since more specific and efficacious second-generation therapies will require improved descriptions of the mechanisms underlying successful pharmacotherapeutic intervention. Once new and effective therapies are developed for NDDs by preclinical studies, designing and executing their clinical trials will require coordinated effort and significant resources from academia, government, private foundations and pharmaceutical companies.

Discussing the neurobiological mechanisms of NDDs, the Special Task Force of the International Stress and Behavior Society (ISBS) has presented recommendations on improving drug discovery, pharmacotherapy and translational research of NDDs. Based on clinical, preclinical and translational models, these recommendations cover multiple areas ranging from methodological considerations to conceptual strategies of future research (Fig. 3). We realize that these recommendations cannot be addressed all in once, and that studies employing experimental animals will always remain an approximation of the human condition. Despite these limitations, increasing our awareness of factors 
relevant for improved understanding of NDDs will advance this field and may trigger innovative crossdisciplinary research that goes beyond traditional methods and concepts.

\section{Declaration of Interest:}

This collaborative study was initiated and coordinated by the International Stress and Behavior Society (ISBS) Task Force on Neurodevelopmental Disorders. SN, MK and HY are supported by Grantsin-Aid for Scientific Research (KAKENHI) of the Japan Society for the Promotion of Science (JSPS. Grants 25282221, 21200017, 25119509 and 15K15404. This research is also supported by Guangdong Ocean University (AVK, CS), ZENEREI Research Center, St. Petersburg State University, the Government of the Russian Federation (Act 211, contract 02-A03.21.0006; AVK), and the Institute of Experimental Medicine RAS (VMK). AVK is the Director of ZENEREI Research Center and President of ISBS. The authors have no other relevant affiliations or financial involvement with any organization or entity with a financial interest in or financial conflict with the subject matter or materials discussed in the manuscript, apart from disclosed. 
Figure 1. Neurodevelopmental disorders (NDDs) and their treatment. Panel A outlines therapeutic approaches to treating NDDs and associated other neuropsychiatric illnesses (see Table 1). ASD - autism spectrum disorder, ADHD - attention deficit hyperactivity disorder, OCD - obsessive compulsive disorder, PTSD - post-traumatic stress disorder. Panel B shows the ontogenetic timeline of key neuronal processes related to neural development, as well as the prevalence and heritability estimates (bottom left) and key behavioral symptoms (bottom right) of NDDs; ID - intellectual disabilities, CD - communication disorders. Panel C shows high comorbidity rates among NDDs and with selected other related disorders (ICD - impulse control disorder). 


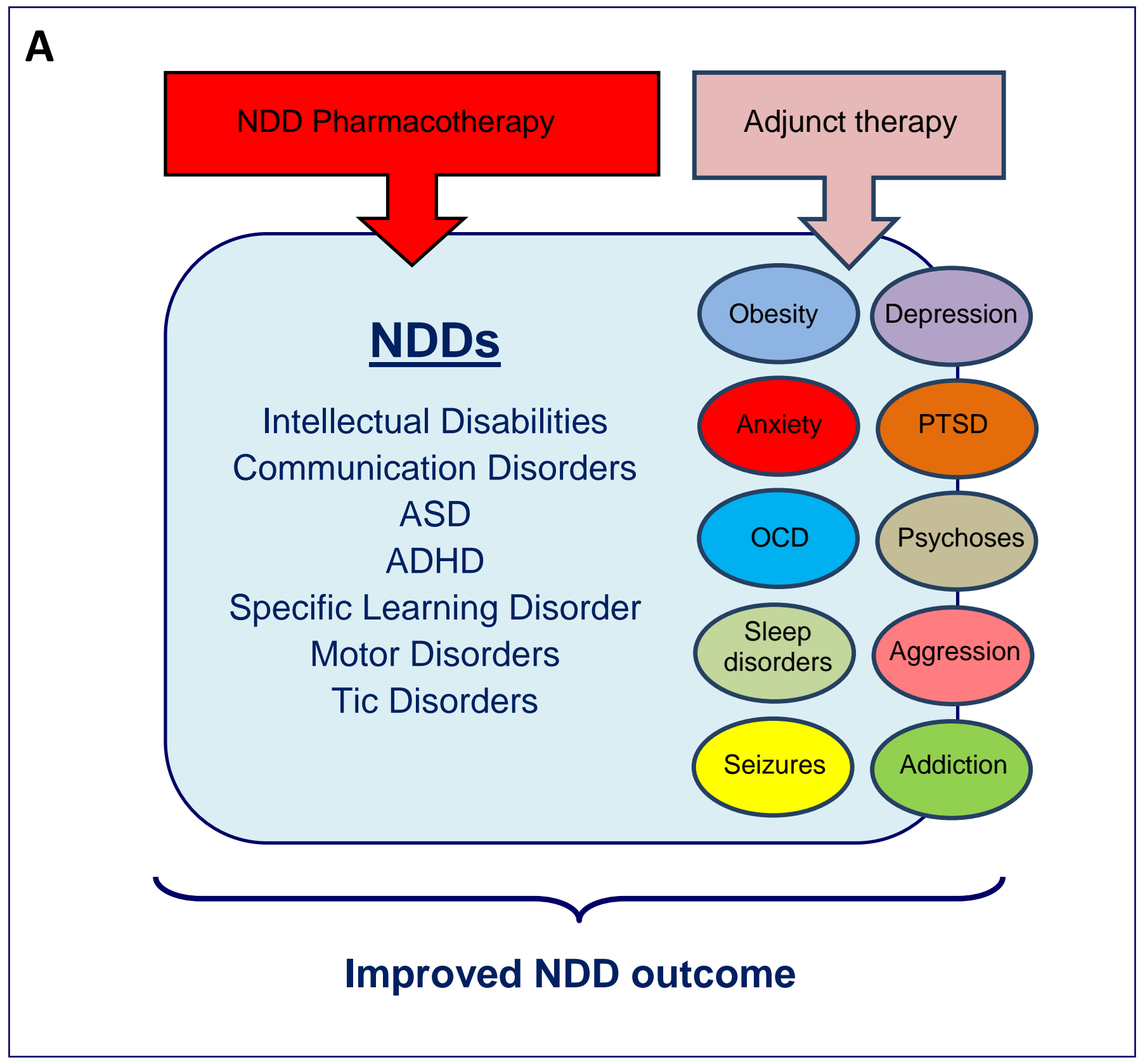




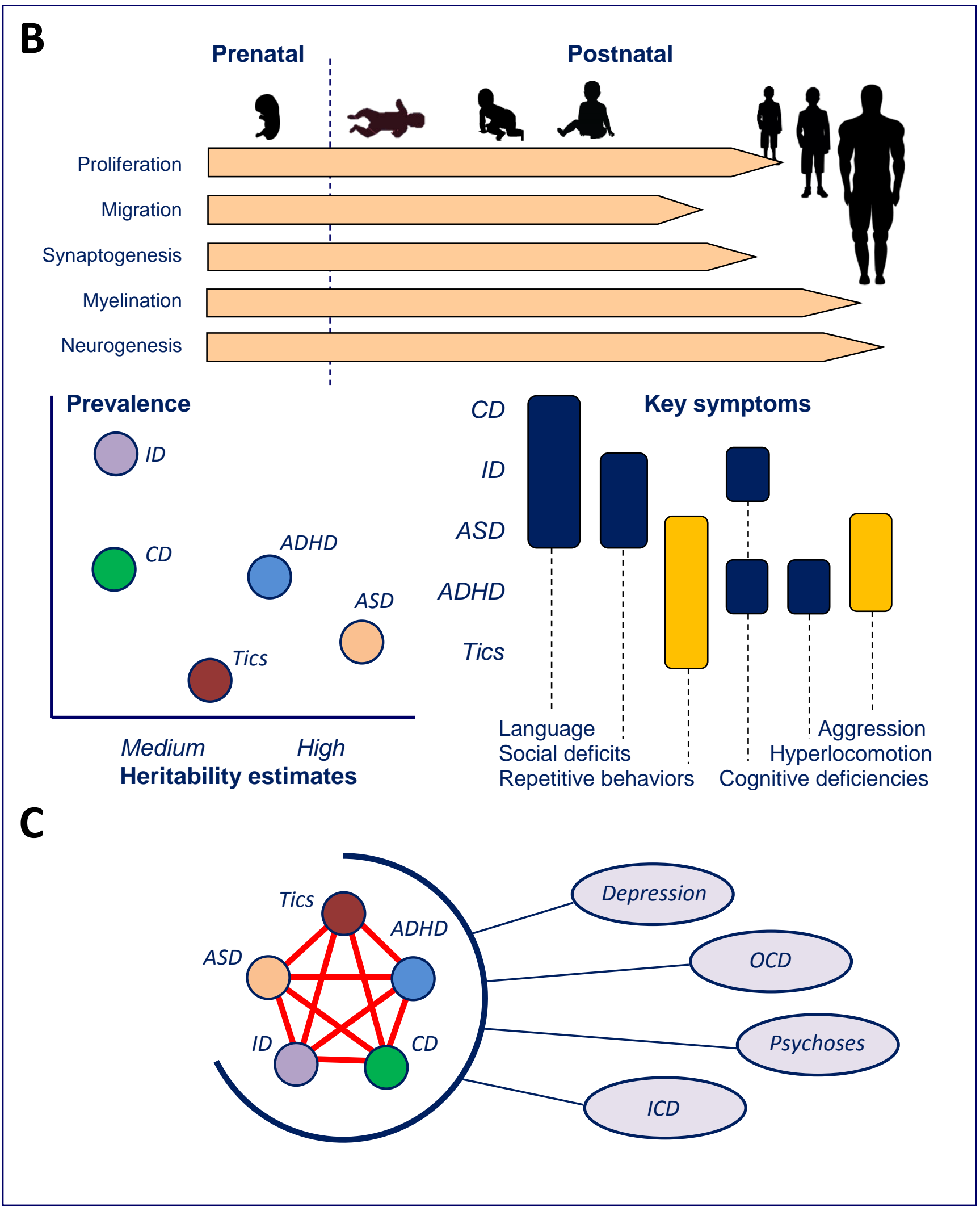


Figure 2. Steps involved in in-vivo development of anti-NDD therapies. Animal size reflects the relative usage of respective species (rodents, chicks, hon-human primates, zebrafish, fruit flies) in NDD research

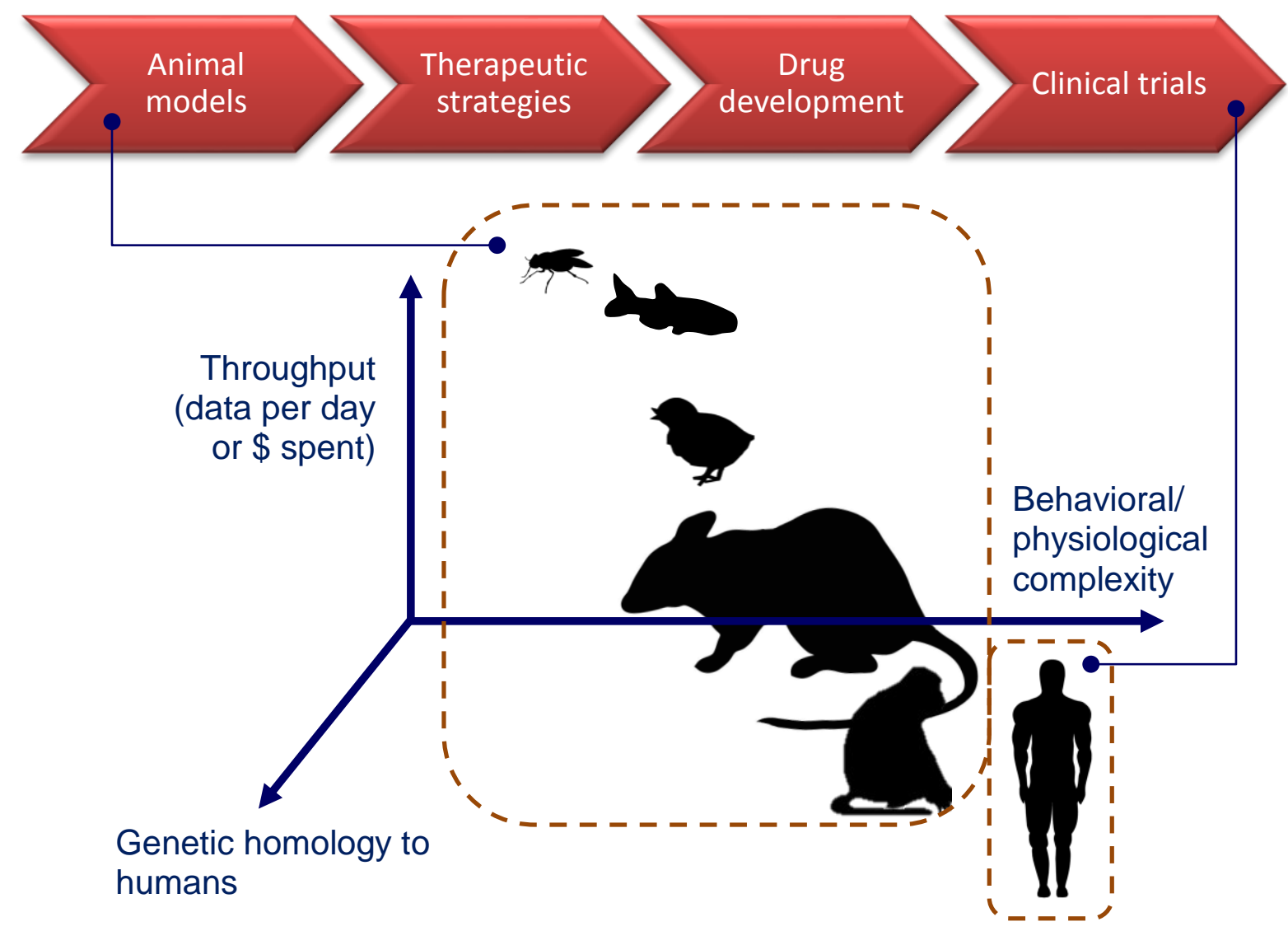


Figure 3. The proposed integrative approach to improving pharmacotherapies of clinical neurodevelopmental disorders (NDDs), based on the recommendations proposed by the ISBS Task Force on NDDs

Use a wider spectrum of drugs with different mechanisms of action

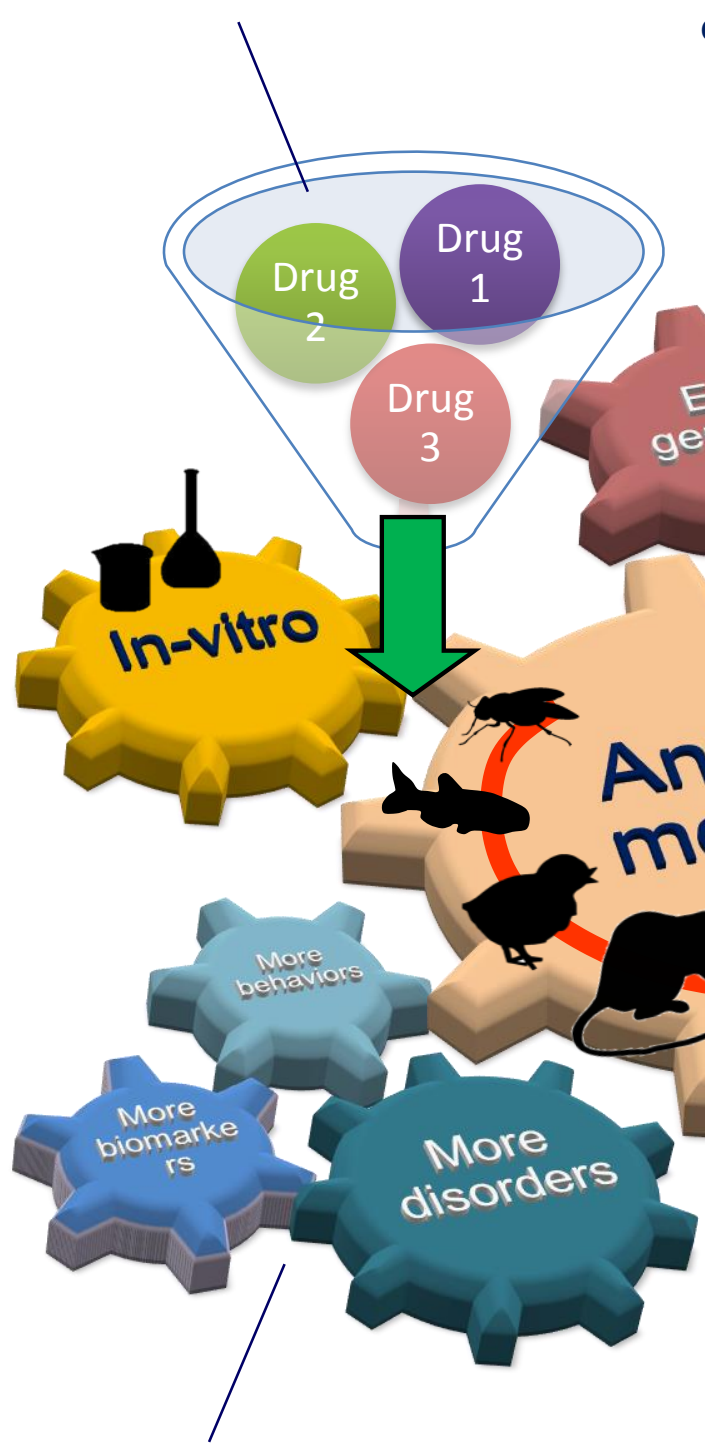

Using more behavioral measures Using more physiological biomarkers Examining a wider spectrum of disorders
Examine a wider range of genetic, epigenetic and environmental factors (e.g., enrichment), gene $x$ environment interactions and G-E correlations 
Table 1. Major groups of neurodevelopmental disorders currently listed in Diagnostic Statistical Manual (DSM-5) ${ }^{1}$

\begin{tabular}{|c|c|c|}
\hline Disorders & Major symptoms & $\begin{array}{l}\text { Availability of } \\
\text { animal models }\end{array}$ \\
\hline Intellectual Disabilities (ID): & $\begin{array}{l}\text { Impaired mental functions in conceptual (language, reading, writing, } \\
\text { knowledge, interpretation), social (empathy, compassion, judgment, } \\
\text { communication, harmony) and practical (personal care, financial } \\
\text { management, hobby) aspects }\end{array}$ & + \\
\hline Communication Disorders & $\begin{array}{l}\text { Difficulties in language, speech, phonetic fluency or social } \\
\text { communication }\end{array}$ & $?^{*}$ \\
\hline $\begin{array}{l}\text { Autism Spectrum Disorder } \\
\text { (ASD): }\end{array}$ & $\begin{array}{l}\text { Persistent deficits in reciprocal social communication and } \\
\text { interaction, as well as restricted, repetitive patterns of behavior, } \\
\text { interests or thoughts }\end{array}$ & + \\
\hline $\begin{array}{l}\text { Attention-Deficit/Hyperactivity } \\
\text { Disorder (ADHD): }\end{array}$ & Impaired attention with bursts of hyperactivity/impulsivity & + \\
\hline Specific Learning Disorder: & Difficulties with learning skills like reading, writing or spelling & Not possible \\
\hline Motor Disorders & $\begin{array}{l}\text { Impaired execution of coordinated motor skills, or repetitive motor } \\
\text { behaviors }\end{array}$ & + \\
\hline Tic Disorders & $\begin{array}{l}\text { Habitual sudden, rapid, recurrent and non-rhythmic motor } \\
\text { movements or vocalizations (including Tourette's syndrome) }\end{array}$ & + \\
\hline
\end{tabular}

* While animals do not have language, their vocalizations (e.g., rodent USVs, bird songs or primate 'calls') may potentially be relevant to modeling neurobiological bases of communication 
Table 2. Selected animal (rodent) models of neurodevelopmental disorders. ID - intellectual disabilities, ASD - Autism Spectrum Disorder, ADHD - Attention Deficit/Hyperactivity Disorder, SLD - Specific Learning Disorder, CD - communication disorders (see Table 1 for details).

\begin{tabular}{|lcccll|}
\hline \multicolumn{1}{|c}{ Rodent models } & \multicolumn{3}{c}{ Availability in other model organisms } & \multicolumn{1}{c}{ Clinical relevance } \\
& Zebrafish & Chicks & Primates & \\
\hline Social/preference tests & + & + & + & Social deficit in ASD \\
Social recognition & + & + & + & Social deficit in ASD, ID \\
Open field test & + & + & + & Hyperactivity in ADHD \\
Various memory tests & + & + & + & Cognitive deficits in ID, SLD and ADHD \\
Various attention tests & + & + & + & Attention deficits in ADHD \\
Various impulsivity tests & + & + & + & Increased impulsivity in ADHD \\
Self-grooming test & & & & & Behavioral perseverations in ASD and tics \\
Aggression test & + & + & + & Aggression in ID, ADHD \\
Marble burying test & & & & Behavioral perseverations in ASD \\
Ultrasonic vocalizations & & & + & + & Social deficits in ASD, ID or CD \\
\hline
\end{tabular}


Table 3. Main drugs currently used to treat major neurodevelopmental disorders (NDDs). ASD autism spectrum disorder, ADHD - attention deficit hyperactivity disorder

\begin{tabular}{|lll|}
\hline Drugs & Main profile and mechanism of action & NDDs treated \\
\hline Risperidone (Risperdal) & Atypical antipsychotic, blocks D2 and 5-HT2A receptors & Tics, ASD*** \\
Haloperidol (Haldol) & Typical antipsychotic (neuroleptic), blocks D2 receptors & Tics* \\
Pimozide (Orap) & Typical antipsychotic (neuroleptic), blocks D2,D3 and D4 receptors & Tics* \\
Clonidine (Catapres) & Sympatolytic $\alpha$ 2 adrenergic- and imidazoline receptor agonist & Tics, ADHD* \\
Aripiprazole (Abilify) & Atypical antipsychotic, partial agonist at dopamine and 5-HT receptors & Tics, ASD*** \\
Atomoxetine (Strattera) & Non-stimulant norepinephrine reuptake inhibitor & ADHD* \\
Methylphenidat (Ritalin) & Stimulant, dopamine-norepinephrine reuptake inhibitor & ADHD* \\
Lisdexamfetamine (Vyvanse) & Stimulant prodrug, blocks monoamine uptake & ADHD* \\
Olanzapine (Zyprexa) & Binds to $\alpha 1$, dopamine, histamine H-1, muscarinic, and 5-HT2 receptors & ASD \\
Ziprasidone (Geodon) & Blocks dopamine/serotonin receptors and monoamine reuptake & ASD \\
Adderall*** & Stimulant amphetamines, block dopamine-norepinephrine reuptake & ADHD* \\
Buspirone (Buspar) & Anxiolytic, serotonin 5-HT1A receptor partial agonist & ADHD, ASD \\
Naltrexone & Antagonist of $\mu$-opioid receptor & ASD \\
SSRIs**** & Antidepressants, selective serotonin reuptake inhibitors & ASD, ADHD \\
\hline
\end{tabular}

* US Federal Drug Administration (FDA)-approved drugs

** Mostly used to treat aggression associated with ASD

*** A combination of amphetamine and dextroamphetamine

**** Mostly used to treat some (e.g., aggression, affective deficits) aspects of NDDs, but can trigger neurodevelopmental deficits in off-spring 
Table 4. Examples of various tests to characterize neural development and behavior of young and adult rodents.

\begin{tabular}{|c|c|}
\hline Category & Tests \\
\hline Simple reflexes & $\begin{array}{l}\text { Sensorimotor (pupillary, salivation, lacrimation) reflexes, acoustic startle*, limb grasping, } \\
\text { somatomotor (loss of crossed-extensor reflex and rooting response, vibrissae response) }\end{array}$ \\
\hline $\begin{array}{l}\text { Sensory and } \\
\text { motor }\end{array}$ & $\begin{array}{l}\text { Cliff avoidance*, olfactory discrimination*, righting, gait analysis, rotarod, grip strength test, } \\
\text { nest building, rope climbing test, vertical screen, self-grooming analyses and the open field test* }\end{array}$ \\
\hline Attention & Various attention tests* \\
\hline Cognitive & $\begin{array}{l}\text { Olfactory conditioning*, T-maze*, Morris water maze, passive and active avoidance tests*, } \\
\text { operant conditioning schedules*, homing and object recognition tests* }\end{array}$ \\
\hline Social & Ultrasonic vocalization, social interaction* and social preference tests* \\
\hline Emotionality & Elevated plus maze, light-dark box*, social interaction test*, self-grooming analysis \\
\hline
\end{tabular}

* Conceptually similar tests are also available for aquatic (zebrafish) models, illustrating evolutionarily conserved trains across species 
Table 5. Summary of selected additional strategies to improve drug development to treat neurodevelopmental pathogenesis

\begin{tabular}{|c|c|}
\hline Strategy & Comments \\
\hline Innovate drug selection and screening & $\begin{array}{l}\text { Use a wider spectrum of compounds, especially those beyond } \\
\text { traditional mechanisms of anti-NDD therapy }\end{array}$ \\
\hline Develop disorder-specific drugs & Drugs targeting ASD vs ADHD or depression \\
\hline Develop sex- and age-specific drugs & For different disorder subtypes and cohorts \\
\hline $\begin{array}{l}\text { Parallel behavioral changes with } \\
\text { electrophysiological biomarkers }\end{array}$ & $\begin{array}{l}\text { Use neuroimaging approaches (e.g., functional magnetic resonance } \\
\text { imaging or mismatch negativity analyses) sensitive to NDDs* }\end{array}$ \\
\hline $\begin{array}{l}\text { Focus on additional disorders and their } \\
\text { comorbidity }\end{array}$ & E.g., with PTSD, aggression, anxiety and psychoses \\
\hline $\begin{array}{l}\text { Focus on 'adult' developmental disorders beyond } \\
\text { DSM-5 }\end{array}$ & E.g., child and adult PTSD and depression \\
\hline $\begin{array}{l}\text { Uncover what NDD phenotypes are shaped by } \\
\text { neurodevelopmental vs. neurochemical changes }\end{array}$ & $\begin{array}{l}\text { Use of conditional transgenic animals and/or prenatal } \\
\text { environmental factors }\end{array}$ \\
\hline $\begin{array}{l}\text { Establish developmental timelines of phenotypes } \\
\text { preceding the full-blown onset of NDDs }\end{array}$ & Apply longitudinal studies \\
\hline Examine gene-environment correlations & $\begin{array}{l}\text { Refine analysis of mother- and father-offspring, as well as early } \\
\text { 'peer-peer' social interactions }\end{array}$ \\
\hline $\begin{array}{l}\text { Assess developmental genomic responses in CNS } \\
\text { in a region-specific manner }\end{array}$ & $\begin{array}{l}\text { Use anatomically comprehensive databases of the developing } \\
\text { human brain, including in situ hybridization, and microarray } \\
\text { analyses** }\end{array}$ \\
\hline $\begin{array}{l}\text { Include more research and evidence generated by } \\
\text { complementary alternative medicine (CAM) }\end{array}$ & $\begin{array}{l}\text { Examine anti-NDD potential of nutrients, natural products and plant } \\
\text { extracts, including those used in traditional Chinese medicine, } \\
\text { Indian Ayurveda and Native/Latin-American or African folk } \\
\text { medicine. }\end{array}$ \\
\hline
\end{tabular}

*See, for example, ${ }^{80,81,101-102}$ for details.

** See ${ }^{102-105}$ for details of NDD-associated susceptibility genes' upregulation in the prefrontal cortex, and prenatal abundance of NDD-related (e.g., ASD-related) gene expression. 


\section{Bibliography:}

Papers of special note have been highlighted as either of interest $\left(^{*}\right)$ or of considerable interest $(* *)$ to readers.

1. American Psychiatric A, American Psychiatric A, Force DSMT. Diagnostic and statistical manual of mental disorders : DSM-5.

2. Hansen RL, Rogers SJ, Publishing AP. Autism and Other Neurodevelopmental Disorders: American Psychiatric Pub., 2013.

3. Ding B. Gene expression in maturing neurons: regulatory mechanisms and related neurodevelopmental disorders. Sheng li xue bao : [Acta physiologica Sinica] 2015;67(2):113-33.

4. Frederick AL, Stanwood GD. Drugs, Biogenic Amine Targets and the Developing Brain.

Developmental neuroscience 2009;31(1-2):7-22.

5. Rice D, Barone S. Critical periods of vulnerability for the developing nervous system: evidence from humans and animal models. Environmental Health Perspectives 2000;108(Suppl 3):511-33.

6. Hu WF, Chahrour MH, Walsh CA. The diverse genetic landscape of neurodevelopmental disorders. Annual review of genomics and human genetics 2014;15:195-213. ** An excellent review of genetic factors underlying NDDs.

7. Ghosh A, Michalon A, Lindemann L, et al. Drug discovery for autism spectrum disorder: challenges and opportunities. Nature reviews Drug discovery 2013;12(10):777-90. ** A timely and comprehensive review of pharmacotherapeutic strategies to treat autism.

8. Gottesman, II, Gould TD. The endophenotype concept in psychiatry: etymology and strategic intentions. The American journal of psychiatry 2003;160(4):636-45.

9. Congdon E, Poldrack RA, Freimer NB. Neurocognitive phenotypes and genetic dissection of disorders of brain and behavior. Neuron 2010;68(2):218-30.

10. Frazer KA, Murray SS, Schork NJ, Topol EJ. Human genetic variation and its contribution to complex traits. Nature reviews Genetics 2009;10(4):241-51. 
11. Mitchell K, Porteus DJ. Rethinking the genetic architecture of schizophrenia. Psychological medicine 2011;41:19-32.

12. Arcos-Burgos M, Jain M, Acosta MT, et al. A common variant of the latrophilin 3 gene, LPHN3, confers susceptibility to ADHD and predicts effectiveness of stimulant medication. Molecular psychiatry 2010;15(11):1053-66.

13. Lange M, Norton W, Coolen M, et al. The ADHD-susceptibility gene lphn3.1 modulates dopaminergic neuron formation and locomotor activity during zebrafish development. Molecular psychiatry 2012; 17(9):946-954. * An interesting translational study using human ADHD susceptibility candidate gene in a zebrafish model of this disorder.

14. Elia J, Gai X, Xie HM, et al. Rare structural variants found in attention-deficit hyperactivity disorder are preferentially associated with neurodevelopmental genes. Molecular psychiatry 2009;15(6):637-46.

15. Lesch KP, Selch S, Renner TJ, et al. Genome-wide copy number variation analysis in attentiondeficit/hyperactivity disorder: association with neuropeptide Y gene dosage in an extended pedigree. Molecular psychiatry 2011;16(5):491-503.

16. Lionel AC, Crosbie J, Barbosa N, et al. Rare copy number variation discovery and cross-disorder comparisons identify risk genes for ADHD. Science translational medicine 2011;3(95):95ra75. 17. Williams NM, Zaharieva I, Martin A, et al. Rare chromosomal deletions and duplications in attention-deficit hyperactivity disorder: a genome-wide analysis. Lancet 2010;376(9750):1401-8. 18. Sebat J, Levy DL, McCarthy SE. Rare structural variants in schizophrenia: one disorder, multiple mutations; one mutation, multiple disorders. Trends Genet 2009;25(12):528-35.

19. Walsh CA. Genetic malformations of the human cerebral cortex. Neuron 1999;23(1):19-29.

20. Walsh CA, Engle EC. Allelic diversity in human developmental neurogenetics: insights into biology and disease. Neuron 2010;68(2):245-53.

21. Porteous DJ, Millar JK, Brandon NJ, Sawa A. DISC1 at 10: connecting psychiatric genetics and neuroscience. Trends in molecular medicine 2011;17(12):699-706. 
22. Molina BS, Pelham WE, Jr. Childhood predictors of adolescent substance use in a longitudinal study of children with ADHD. Journal of abnormal psychology 2003;112(3):497-507.

23. Grace AA. Dopamine system dysregulation by the ventral subiculum as the common pathophysiological basis for schizophrenia psychosis, psychostimulant abuse, and stress. Neurotoxicity research 2010;18(3-4):367-76.

24. Leonard JR, Klocke BJ, D'Sa C, et al. Strain-dependent neurodevelopmental abnormalities in caspase-3-deficient mice. J Neuropathol Exp Neurol 2002;61(8):673-7.

25. Mitchell K. The miswired brain: making connections from neurodevelopment to psychopathology. BMC Biology 2011;9(23).

26. Thornton GK, Woods CG. Primary microcephaly: do all roads lead to Rome? Trends Genet 2009;25(11):501-10.

27. Valiente M, Marin O. Neuronal migration mechanisms in development and disease. Current opinion in neurobiology 2010;20(1):68-78.

28. Waddington $\mathrm{CH}$. The strategy of genes. 1957.

29. Lisman JE, Coyle JT, Green RW, et al. Circuit-based framework for understanding neurotransmitter and risk gene interactions in schizophrenia. Trends in neurosciences 2008;31(5):234-42.

30. Birnbaum R, Jaffe AE, Hyde TM, et al. Prenatal expression patterns of genes associated with neuropsychiatric disorders. The American journal of psychiatry 2014;171(7):758-67. ** An important study showing the link between common neuropsychiatric disorders and upregulated prenatal gene expression, implicating neurodevelopmental trajectories in brain deficits, including common NDDs, such as ID and ASD.

31. Mazin P, Xiong J, Liu X, Yan Z, et al. Widespread splicing changes in human brain development and aging. Molecular systems biology 2013;9:633.

32. Lambert N, Wermenbol V, Pichon B, et al. A familial heterozygous null mutation of MET in autism spectrum disorder. Autism research 2014;7(5):617-22.

33. Gould TD, Gottesman, II. Psychiatric endophenotypes and the development of valid animal models. 
Genes Brain Behav 2006;5(2):113-9.

34. Jones LJ, Norton WH. Using zebrafish to uncover the genetic and neural basis of aggression, a frequent comorbid symptom of psychiatric disorders. Behavioural brain research 2015;276:171-80. 35. Arnedo J, Svrakic DM, Del Val C, et al. Uncovering the Hidden Risk Architecture of the Schizophrenias: Confirmation in Three Independent Genome-Wide Association Studies. The American journal of psychiatry 2015;172(2):139-53. * An important study on the emerging 'bigger' complexity of neuropsychiatric disorders.

36. Robison A, Vialou V, Sun H-S, et al. Fluoxetine epigenetically alters the CaMKII $\alpha$ promoter in nucleus accumbens to regulate $\Delta$ FosB binding and antidepressant effects. Neuropsychopharmacology 2014;39(5):1178-86.

37. Sweatt JD. Experience-dependent epigenetic modifications in the central nervous system. Biological psychiatry 2009;65(3):191-97.

38. Kalueff AV, Olivier JD, Nonkes LJ, et al. Conserved role for the serotonin transporter gene in rat and mouse neurobehavioral endophenotypes. Neurosci Biobehav Rev 2010;34(3):373-86. * A timely discussion on the role of serotonin and serotonin transporter in translational models of brain disorders, including NDDs.

39. Altieri SC, Yang H, O'Brien HJ, et al. Perinatal vs Genetic Programming of Serotonin States Associated with Anxiety. Neuropsychopharmacology 2014, in press.

40. Kepser LJ, Homberg JR. The neurodevelopmental effects of serotonin: a behavioural perspective. Behavioural brain research 2015;277:3-13. * An important discussion of developmental, neurotrophic role of serotonin, highly relevant to $N D D$ s.

41. Croen LA, Grether JK, Yoshida CK, et al. Antidepressant use during pregnancy and childhood autism spectrum disorders. Archives of general psychiatry 2011;68(11):1104-12.

42. El Marroun H, White TJ, van der Knaap NJ, et al. Prenatal exposure to selective serotonin reuptake inhibitors and social responsiveness symptoms of autism: population-based study of young children. The British journal of psychiatry 2014;205(2):95-102. 
43. Clayton J, Collins F. Policy: NIH to balance sex in cell and animal studies. Nature 2014;509:28283.

44. Stewart AM, Kalueff AV. Anxiolytic drug discovery: what are the novel approaches and how can we improve them? Expert Opin Drug Discov 2014;9(1):15-26.

45. Holmes EA, Craske MG, Graybiel AM. Psychological treatments: A call for mental-health science. Nature 2014;511(7509):287-89.

46. Kalueff AV, Echevarria DJ, Stewart AM. Gaining translational momentum: more zebrafish models for neuroscience research. Prog Neuropsychopharmacol Biol Psychiatry 2014;55:1-6.

47. Kalueff AV, Stewart AM, Gerlai R. Zebrafish as an emerging model for studying complex brain disorders. Trends Pharmacol Sci 2014;35(2):63-75.

48. Kalueff AV, Gebhardt M, Stewart AM, et al. Towards a comprehensive catalog of zebrafish behavior 1.0 and beyond. Zebrafish 2013;10(1):70-86.

49. Stewart AM, Nguyen M, Wong K, et al. Developing zebrafish models of autism spectrum disorder (ASD). Prog Neuropsychopharmacol Biol Psychiatry 2014;50:27-36.

50. Lange M, Norton W, Coolen M, et al. The ADHD-linked gene Lphn3.1 controls locomotor activity and impulsivity in zebrafish. Mol Psychiatry 2012;17(9):855.

51. Lange M, Norton W, Coolen M, et al. The ADHD-susceptibility gene lphn3.1 modulates dopaminergic neuron formation and locomotor activity during zebrafish development. Mol Psychiatry 2012;17(9):946-54.

52. Koshiba M, Karino G, Senoo A, et al. Peer attachment formation by systemic redox regulation with social training after a sensitive period. Sci Rep 2013;3:2503.

53. Shirakawa YN, Koshiba, M. Peer-Social Network Development Revealed by the Brain Multivariate Correlation Map with 10 Monoamines and 11 Behaviors. Journal of Clinical Toxicology

2013;3(2):1000161. ** An important translational 'integrative’ study linking animal social behaviors/learning to brain neurochemical phenotypes.

54. Koshiba MN, Mimura, K.; Senoo, A., et al. Socio-emotional development evaluated by Behaviour 
Output analysis for Quantitative Emotional State Translation: towards early diagnosis of individuals with developmental disorders. OA Autism 2013;1(2):18.

55. Koshiba M, Mimura K, Sugiura Y, et al. Reading marmoset behavior 'semantics' under particular social context by multi-parameters correlation analysis. Progress in neuro-psychopharmacology \& biological psychiatry 2011;35(6):1499-504.

56. Koshiba M, Senoo A, Mimura K, et al. A cross-species socio-emotional behaviour development revealed by a multivariate analysis. Scientific Reports $2013 ; 3$.

57. Kaur K, Simon AF, Chauhan V, Chauhan A. Effect of bisphenol A on Drosophila melanogaster behavior - a new model for the studies on neurodevelopmental disorders. Behav Brain Res $2015 ; 284: 77-84$

58. Wise A, Tenezaca L, Fernandez RW, et al. Drosophila mutants of the autism candidate gene neurobeachin (rugose) exhibit neuro-developmental disorders, aberrant synaptic properties, altered locomotion, and impaired adult social behavior and activity patterns. J Neurogenet 2015; 14:1-9. 59. van der Voet M, Harich B, et al. ADHD-associated dopamine transporter, latrophilin and neurofibromin share a dopamine-related locomotor signature in Drosophila. Mol Psychiatry 2015, in press.

60. Ickes BR, Pham TM, Sanders LA, et al. Long-term environmental enrichment leads to regional increases in neurotrophin levels in rat brain. Experimental neurology 2000;164(1):45-52.

61. Mohammed AH, Henriksson BG, Söderström S, et al. Environmental influences on the central nervous system and their implications for the aging rat. Behavioural brain research 1993;57(2):183-91. 62. Schneider T, Turczak J, Przewłocki R. Environmental enrichment reverses behavioral alterations in rats prenatally exposed to valproic acid: issues for a therapeutic approach in autism. Neuropsychopharmacology 2006;31(1):36-46.

63. Hannigan JH, Berman RF, Zajac CS. Environmental enrichment and the behavioral effects of prenatal exposure to alcohol in rats. Neurotoxicology and teratology 1993;15(4):261-66.

64. Berman RF, Hannigan JH, Sperry MA, Zajac CS. Prenatal alcohol exposure and the effects of 
environmental enrichment on hippocampal dendritic spine density. Alcohol 1996;13(2):209-16.

65. Wainwright P, Levesque S, Krempulec L, et al. Effects of environmental enrichment on cortical depth and Morris-maze performance in B6D2F 2 mice exposed prenatally to ethanol. Neurotoxicology and teratology 1993;15(1):11-20.

66. Pamplona FA, Pandolfo P, Savoldi R, et al. Environmental enrichment improves cognitive deficits in spontaneously hypertensive rats (SHR): relevance for attention deficit/hyperactivity disorder (ADHD). Progress in Neuro-Psychopharmacology and Biological Psychiatry 2009;33(7):1153-60. 67. Del Arco A, Segovia G, Canales J, et al. Environmental enrichment reduces the function of D1 dopamine receptors in the prefrontal cortex of the rat. Journal of neural transmission 2007;114:43-48. 68. Perry JL, Stairs DJ, Bardo MT. Impulsive choice and environmental enrichment: effects of damphetamine and methylphenidate. Behavioural brain research 2008;193(1):48-54.

69. Jaffee SR, Price TS. Gene-environment correlations: a review of the evidence and implications for prevention of mental illness. Molecular psychiatry 2007;12(5):432-42.

70. Ullmann JFP, Cowin G, Kurniawan ND, Collin SP. A three-dimensional digital atlas of the zebrafish brain NeuroImage 2010;51(1):76-82.

71. Ullmann JFP, Cowin G, Collin SP. Quantitative assessment of brain volumes in fish: comparison of methodologies. Brain Behavior and Evolution 2010;76(261-270).

72. Ullmann JF, Watson C, Janke AL, et al. An MRI atlas of the mouse basal ganglia. Brain structure \& function 2014;219(4):1343-53.

73. Richards KL, Kurniawan ND, Yang Z, et al. Hippocampal volume and cell density changes in a mouse model of human genetic epilepsy. Neurology 2013;80(13):1240-46.

74. Calabrese E, Johnson GA, Watson C. An ontology-based segmentation scheme for tracking postnatal changes in the developing rodent brain with MRI. NeuroImage 2013;67:375-84.

75. Calabrese E, Johnson GA. Diffusion tensor magnetic resonance histology reveals microstructural changes in the developing rat brain. NeuroImage 2013;79:329-39.

76. Olson LE, Roper RJ, Baxter LL, et al. Down syndrome mouse models Ts65Dn, Ts1Cje, and 
Ms1Cje/Ts65Dn exhibit variable severity of cerebellar phenotypes. Developmental dynamics : an official publication of the American Association of Anatomists 2004;230(3):581-9.

77. Malkova NV, Gallagher JJ, Yu CZ, et al. Manganese-enhanced magnetic resonance imaging reveals increased DOI-induced brain activity in a mouse model of schizophrenia. Proceedings of the National Academy of Sciences of the United States of America 2014;111(24):E2492-500.

78. Inoue T, Majid T, Pautler RG. Manganese enhanced MRI (MEMRI): neurophysiological applications. Reviews in Neurosciences 2011;22(6):675-94.

79. Zhang X, Bearer EL, Boulat B, et al. Altered neurocircuitry in the dopamine transporter knockout mouse brain. PloS one 2010;5(7):e11506.

80. Casey BJ, Oliveri ME, Insel T. A neurodevelopmental perspective on the research domain criteria (RDoC) framework. Biol Psychiatry 2014;76:350-3. ** An important summary of the research domain criteria (RDoCs) concept that targets the cross-disorder nosology (rather than specific symptoms) of psychiatric disorders.

81. Sforazzini F, Bertero A, Dodero L, et al. Altered functional connectivity networks in acallosal and socially impaired BTBR mice. Brain Struct Funct 2014, in press.

82. Allsop SA, Vander Weele CM, Wichmann R, et al. Optogenetic insights on the relationship between anxiety-related behaviors and social deficits. Front Behav Neurosci 2014;8:241.

83. Yizhar O. Optogenetic insights into social behavior function. Biological psychiatry 2012;71(12):1075-80.

84. Tye KM, Deisseroth K. Optogenetic investigation of neural circuits underlying brain disease in animal models. Nature reviews Neuroscience 2012;13(4):251-66.

85. Wong DF, Tauscher J, Grunder G. The role of imaging in proof of concept for CNS drug discovery and development. Neuropsychopharmacology : official publication of the American College of Neuropsychopharmacology 2009;34(1):187-203.

86. Beckmann N, Kneuer R, Gremlich HU, et al. In vivo mouse imaging and spectroscopy in drug discovery. NMR in biomedicine 2007;20(3):154-85. 
87. Chung K, Wallace J, Kim SY, et al. Structural and molecular interrogation of intact biological systems. Nature 2013;497(7449):332-7.

88. Onnink AM, Zwiers MP, Hoogman M, et al. Deviant white matter structure in adults with attentiondeficit/hyperactivity disorder points to aberrant myelination and affects neuropsychological performance. Prog Neuropsychopharmacol Biol Psychiatry 2015;63:14-22.

89. Chiang HL, Chen YJ, Lo YC, et al. Altered white matter tract property related to impaired focused attention, sustained attention, cognitive impulsivity and vigilance in attention-deficit/ hyperactivity disorder. J Psychiatry Neurosci 2015;40(2):140106. *An interesting study showing potential neuroanatomical white matter-related biomarkers of NDDs.

90. Bremner JD, Vythilingam M, Vermetten E, et al. MRI and PET study of deficits in hippocampal structure and function in women with childhood sexual abuse and posttraumatic stress disorder. Am J Psychiatry 2003;160(5):924-32.

91. Bremner JD. Long-term effects of childhood abuse on brain and neurobiology. Child Adolesc Psychiatr Clin N Am 2003;12(2):271-92.

92. Asnis GM, Kohn SR, Henderson M, Brown NL. SSRIs versus non-SSRIs in post-traumatic stress disorder: an update with recommendations. Drugs 2004;64(4):383-404.

93. Stein MB, Kline NA, Matloff JL. Adjunctive olanzapine for SSRI-resistant combat-related PTSD: a double-blind, placebo-controlled study. Am J Psychiatry 2002;159(10):1777-9.

94. Southwick SM, Morgan CA, Charney DS, High JR. Yohimbine use in a natural setting: effects on posttraumatic stress disorder. Biol Psychiatry 1999;46(3):442-4.

95. Pitman RK, Sanders KM, Zusman RM, et al. Pilot study of secondary prevention of posttraumatic stress disorder with propranolol. Biol Psychiatry 2002;51(2):189-92.

96. Zoladz PR, Park CR, Munoz C, Fleshner M, Diamond DM. Tianeptine: an antidepressant with memory-protective properties. Curr Neuropharmacol 2008; 6(4):311-21.

97. Stewart AM, Kalueff AV. Developing better and more valid animal models of brain disorders. Behav Brain Res 2015;276:28-31. 
98. Kalueff AV, Murphy DL. The importance of cognitive phenotypes in experimental modeling of animal anxiety and depression. Neural Plast 2007;2007:52087.

99. Kalueff AV, Wheaton M, Murphy DL. What's wrong with my mouse model? Advances and strategies in animal modeling of anxiety and depression. Behav Brain Res 2007;179(1):1-18.

100. Sullivan PF. Puzzling over schizophrenia: schizophrenia as a pathway disease. Nat Med 2012; $18(2): 210-211$

101. Ceponiene R, Lepistö T, Shestakova A, et al. Speech-sound-selective auditory impairment in children with autism: they can perceive but do not attend. Proc Natl Acad Sci U S A. 2003;100(9):5567-72.

102. Miller JA, Ding S-L, Sunkin SM, et al. Transcriptional landscape of the prenatal human brain. Nature 2014; 508, 199-206. ** An outstanding, anatomically comprehensive atlas of the mid-gestational human brain, including in situ hybridization, high-resolution magnetic resonance imaging and microarray analyses.

103. Stefansson H, Meyer-Lindenberg A, Steinberg S, et al. CNVs conferring risk of autism or schizophrenia affect cognition in controls. Nature 2014;505(7483):361-366.

104. Bourgeron T. From the genetic architecture to synaptic plasticity in autism spectrum disorder. Nat Rev Neurosci. 2015;16(9):551-563.

105. Jaffe AE, Shin J, Collado-Torres L, et al. Developmental regulation of human cortex transcription and its clinical relevance at single base resolution. Nat Neurosci. 2015;18(1):154-161. * An important recent study showing conserved molecular signatures of transcriptional dynamics across brain development, with potential high clinical relevance. 